\title{
Jet Flow Focusing by Corona Discharge for Fluidic Application
}

\author{
Tung Thanh Bui ${ }^{1}$, Thien Xuan Dinh ${ }^{2}$, Tibor Terebessy ${ }^{3}$, Trinh Chu Duc ${ }^{1}$, and Van Thanh Dau ${ }^{4}$ \\ ${ }^{1}$ University of Engineering and Technology, Vietnam National University, Hanoi, Vietnam (tungbt@vnu.edu.vn) \\ ${ }^{2}$ Graduate School of Science and Engineering, Ritsumeikan University, Shiga 525-8577, Japan (thien@cfd.ritsumei.ac.jp) \\ ${ }^{3}$ Atrium Innovation Ltd., Lupton Road, OX10 9BT, Wallingford, United Kingdom (tibor.terebessy@clearviewtraffic.com) \\ ${ }^{4}$ Research Group (Environmental Health), Sumitomo Chemical. Ltd, Hyogo 665-8555, Japan (dauthanhvan@gmail.com)
}

\begin{abstract}
This paper presents a study on a jet flow assisted by low net charge ion wind generated from bipolar corona setup, adding momentum to the bulk flow directed alongside the electrodes and focused in the middle of interelectrode space. It was confirmed that with only $27.1 \mathrm{~mW}$ of consumed discharge power, the corona helped to focus and increased the flow peak velocity up to $71.6 \%$, i.e., from $1.41 \mathrm{~m} / \mathrm{s}$ to $2.42 \mathrm{~m} / \mathrm{s}$. Because the generated air flow remains neutral, this work can contribute to the development of multi-axis fluidic inertial sensors, fluidic mixing and analysis with space constraints and/or where neutralized discharge process is required.
\end{abstract}

Keywords- External flow; Blower; Neutralized ion wind; OpenFOAM; Bipolar corona discharge

\section{INTRODUCTION}

Jet flow in pumping technology is principally created by the interactions of counter-rotating vortex pairs at the edge of an orifice by the oscillating diaphragm in a sealed cavity [1]. The potential applications for the integration of micro devices for synthetic jets cover various research topics such as microelectronics cooling [2], gas monitoring [3], [4], drug delivery [5], inertial sensing [6], [7] and biology research [8]. One of the most common air jet micro generators is based on the piezoelectric lead zirconate titanate (PZT) diaphragm, which has advantages over the conventional fan/blower due to the minimum required volume, reduced power consumption, longer lifetime, and high output power density [9]. Another way to create air jet flow is by electrokinetic actuation, which has recently attracted more interest. Typically the electric field in these generators is provided by corona discharge, which has distinguishable advantages due to the absence of moving parts, small size and reduced weight, low acoustic noise, simple design and easy operation. Among the wide range of applications, the enhancement of heat transfer (spot cooling) and ion wind generators are the most popular [10]. In case of ion wind blowers, both electrodes are placed inside the device and the flow is aimed at an external target.

In this paper, we present the study of a bipolar corona assisted bulk flow for jet generator. The air flow is validated by the integrated thermal sensing elements (hotwires) implemented at several positions along the downstream channel, as well as by an external reference flow sensor. The simulation is conducted in an open-source code environment, OpenFOAM.

\section{CONCEPT DESIGN AND WORKING PRINCIPLE}

The schematic view of working principle is shown in Fig 1. The bulk jet flow is created by a bulk flow generator and the corona discharge is generated by two parallel discharge electrodes placed on both sides of the flow and pointed downstream. The discharge electrodes are cylindrical pins with very small tips to ignite corona discharge and minimize the impact of the electrode itself on the jet flow.

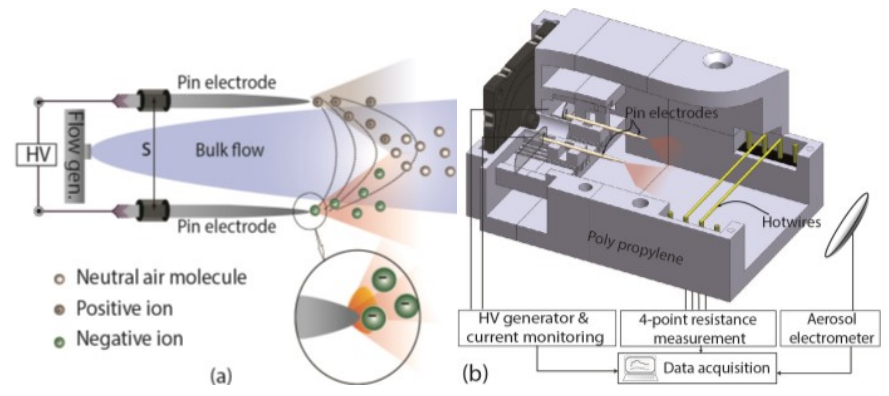

Fig.1 Schematic description of working principle of corona assisted jet flow device (a) and schematic view of measurement setup (b)

In general, an ion wind generator is designed with a $\mathrm{pin} /$ needle/wire as a corona electrode and a plane/ring/grid as the collector electrode. Unipolar ion wind is generated at the corona electrode and yields high velocity near the surface of the counter electrodes, where the charge has to be neutralized. This configuration is not suitable to assist externally generated jet flow due to the positioning of the collector electrode. In order not to impede the flow, the collector electrode needs to be placed off center, or in case of a ring type electrode shown, the electrode diameter needs to exceed the cross sectional dimension of the flow. This however creates a defocusing effect, as the charged particles in the generated ion wind tend to move towards the collector electrode. In addition, such configuration usually leaves residual charge in the air flow, therefore in some applications vortex stream is needed to help with focusing the flow [11]. In our configuration, the two electrodes of opposite polarity are placed in parallel, and generate oppositely charged flows from a single power source ensuring both minimized impediment and focusing of the external jet flow [12]-[14].

The ion wind in our design is simultaneously generated by both pins. The pin tip can be modelled as a protruding hemisphere with extremely high curvature attached to the pin body, which focuses the electric field outwards and nearly parallel to the pin axis. Thus, after being generated at the 
vicinity of tips, ion clouds gain an initial momentum to move in the direction away from the pin tips and in parallel with the electrodes (inset in Fig. 1). Under the impact of the electric field between two electrodes, the clouds of oppositely charged ions from two electrodes impinge at the electrode interspace, preventing them from reaching the counter electrodes. Due to high speed of ion wind and its forwarding momentum, the bulk of ions moves forward, resulting in net flow. This movement of ion wind helps to boost the jet flow in magnitude while keeping the velocity profile maintain the jet flow characteristics.

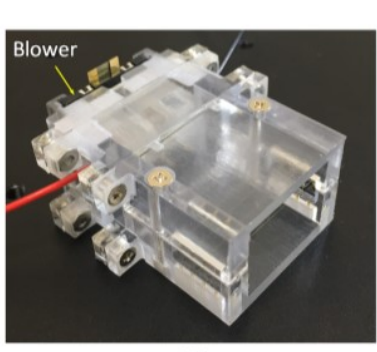

(a)

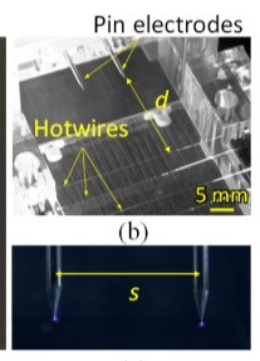

(c)
Fig.2. Fabricated device. Overall view (a), inside the device (b) and device in operation with bipolar discharge (c).

Figure $1 \mathrm{~b}$ shows the schematics of our experimental setup. The actual prototype device (Fig. 2a) has been made from polypropylene with internal cross section of $15 \times 20 \mathrm{~mm}^{2}$ (height $\times$ width). The pin electrodes are aligned and positioned at one end of the device (Fig. 2b, c). The bulk flow is generated by conventional generator assembled at the back of the device. A single direct current high-voltage generator, battery operated, capable of generating $10.0 \mathrm{kV}$ is connected to the pins. The discharge current is recorded at the negative electrode by a precision measuring circuit, which is integrated in the high voltage generator. The system is calibrated with high voltage generator and high voltage meter (Japan Finechem).

The flow generated in the device is measured by an array of 4 hotwires placed across the downstream channel starting from a distance of $12.5 \mathrm{~mm}$ from electrode tips and is aligned in the plane of the discharge electrodes. The spacing between the hotwires is $2.5 \mathrm{~mm}$. The hotwire, made of gold, is bonded to the electric stands embedded in the device's body for signal reading. The hotwire has a diameter of $25.0 \mu \mathrm{m}$ and length of $24.0 \mathrm{~mm}$, its resistance and temperature coefficient of resistance (TCR) are measured as $1.35 \Omega$ and $3700 \mathrm{ppm} /{ }^{\circ} \mathrm{C}$, respectively.

\section{NUMERICAL SIMULATION}

The corona drift region is governed by the Poisson and current continuity equations, which could not be globally solved until now. In this part, to avoid the complications of modeling of the discharge itself, we deploy multi physics simulation to analyze the flow characteristics of our system by treating the corona as a boundary condition [15].

The simulation domain was shown in Fig. 3. For the electric field, voltage was applied to the boundary of the electrodes and the Neumann condition was applied at the edges of the domain. The continuity of the positive/negative current density is described by the ion recombination. At the electrode, we assumed that the corona discharge maintained a constant ion density which is calculated by dividing the discharge current with the surface area of the pin tips $\rho_{ \pm}=I /\left(\mu E_{w} A\right)$, where $A$ is the total area of the tip. The electric field at the wall tip $E_{w}$ is determined based on Peek's law for air as $E_{w}=31(\mathrm{kV} /$ $\mathrm{cm})\left[1+0.308 /(0.5 R)^{1 / 2}\right], R$ is the radius of the tips in units of centimeters.

For the fluidic field, the non-slip condition was set on the pin electrode, the channel and the inlet channel walls. The free condition was used for the outlet and free inlet. The uniform velocity $\mathrm{U}_{\mathrm{b}}$ at the inlet was treated as input parameter and the simulated velocity profile at the hotwire plane was exported to further calculate the average flow velocity by a C-code routine, which convert flow velocity to output voltage on the hotwire. The calculation process considers infinitesimal hotwire elements on which the flow velocity is constant and its value is taken from the obtained simulation profile. This procedure gives very good match with the experimental values [16], [17] [18].

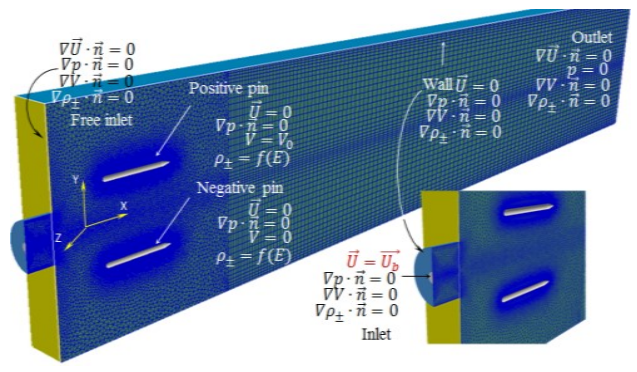

Fig. 3 Meshing and boundary conditions for numerical setup of device. The inlet condition $U_{b}$ is iterated to calibrate the bulk flow generator.

\section{RESULTS AND DISCUSSION}

Figure $4 \mathrm{a}$ shows the voltage $V_{h w}$ measured on the embedded hotwires for different bulk flow speeds generated by the flow generator with voltage applied to its PZT diaphragm in the range of $V_{b}=5.0 \mathrm{~V}$ to $V_{b}=11.0 \mathrm{~V}$. The extrapolation was found to be in good agreement $(<6.8 \%)$ with the experimentally obtained hotwire voltage values for the entire driving voltage range. Fig. $4 \mathrm{~b}$ shows both the simulation result and the measured hotwire output for all hotwire positions and two different driving voltages, $8.0 \mathrm{~V}$ and $11.0 \mathrm{~V}$, respectively.

Figure 5 presents the simulated result of the flow field inside the device where the bulk flow is simulated simultaneously with corona discharge. For the mixing of charge from ion wind, it was confirmed by the aerosol electrometer at the outlet of device that the total charge of the ion wind outside the wind collector is very low and in the order of background noise, e.g several fAs. Since this net charge of ion wind is very small compared with the discharge current (of the order of several $\mu \mathrm{As}$, which is nine orders larger), this confirms that the positive and negative charges are well balanced.

Figure 6 shows the output on hotwire1 when the corona is discharged at different voltages $V_{c}$ from $4.0 \mathrm{kV}$ to $5.25 \mathrm{kV}$ and the driving voltage $V_{b}$ from $5.0 \mathrm{~V}$ to $11.0 \mathrm{~V}$. As it can be seen by the increase of the total voltage on the hotwire, the corona discharge itself has added a considerable momentum to the flow. 


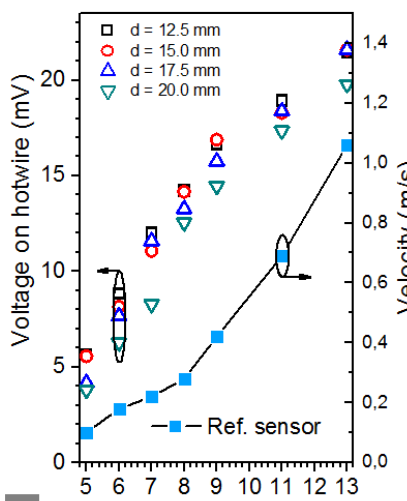

Applied voltage on blower (V)

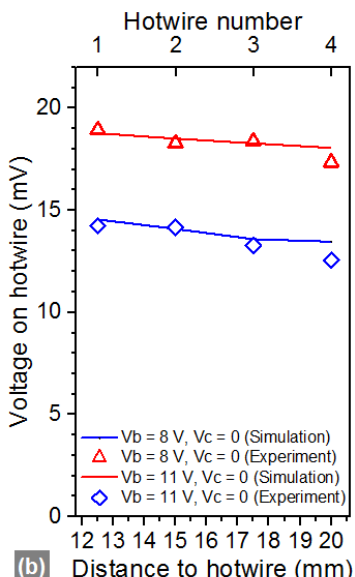

Distance to hotwire $(\mathrm{mm})$

Fig. 4 Hotwire voltage and flow speed vs. applied voltage on bulk flow generator (a) and comparison of simulated results with the experimentally obtained hotwire voltage values for bulk flow driving voltage $8.0 \mathrm{~V}$ and 11.0 $\mathrm{V}(\mathrm{b})$.

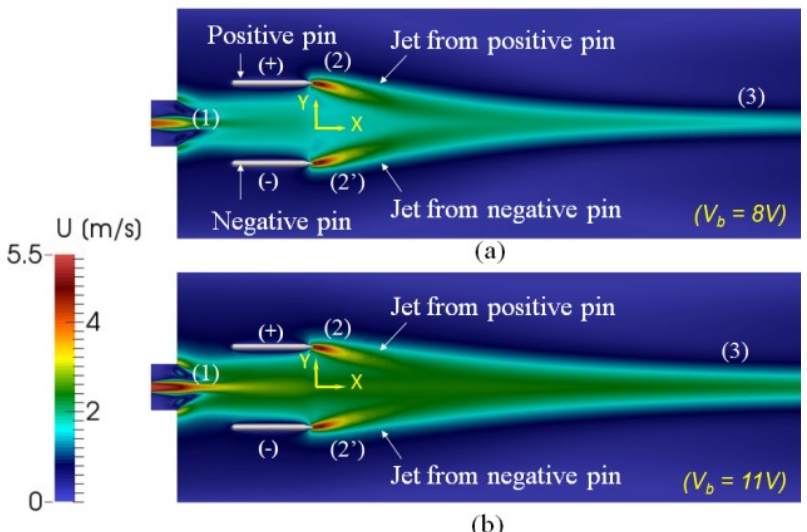

(b)

Fig.5 Flow field inside the device showing bulk flow from generator (1) in the middle and ionic jets from two electrode (2) and (2'). The bulk flow is generated with driving voltage of $8.0 \mathrm{~V} \mathrm{(a)}$ and $11.0 \mathrm{~V}(\mathrm{~b})$, and the corona is discharged at $5.0 \mathrm{kV}$ and $5.78 \mu \mathrm{A}$ and $5.41 \mu \mathrm{A}$, respectively.
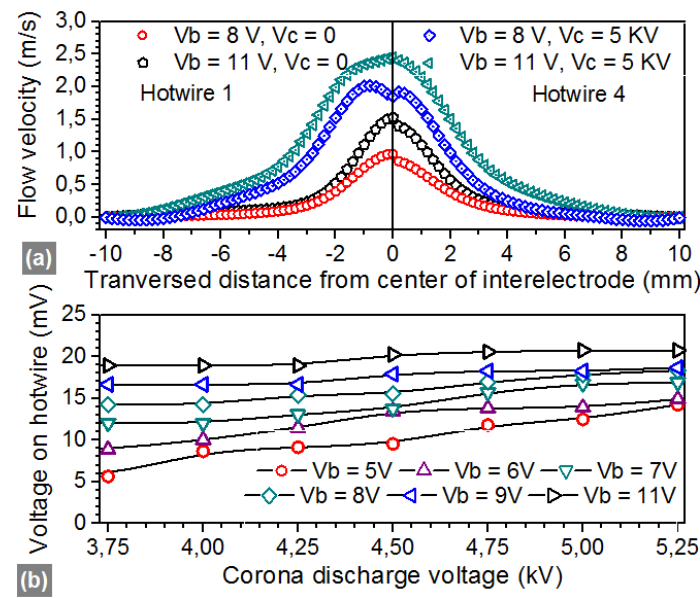

Fig. 6 Effect of corona discharge on bulk flow. (a) Output on hotwire1 versus discharge voltage, corresponding to each applied voltage on bulk flow generator, (b) Velocity profiles at hotwire 1 and hotwire 4, shown for driving voltage set to $8.0 \mathrm{~V}$ and $11.0 \mathrm{~V}$, both with $(5.0 \mathrm{kV}$ discharge voltage $)$ and without corona discharge. The effect of corona is clearly demonstrated by the significant increase of peak velocity.

The profiles of the velocity at hotwire locations 1 and 4 are plotted in each half of Fig. 6a. It is evident that the profiles are significantly enhanced by the existence of corona discharge. The tails of these profiles have negative values, which show that there is a circulatory flow in the channel. It is worth noting that the power for corona discharge itself is small, around 27 $\mathrm{mW}$ and the power consumption in our electric circuit is less than $70 \mathrm{~mW}$ for the experimental condition in Fig. 6b. For reference, the bulk flow generator consumes more than 250 $\mathrm{mW}$ for typical performance.

\section{REFERENCES}

[1] K. Tanaka, V. T. Dau, R. Sakamoto, T. X. Dinh, D. V. Dao, and S. Sugiyama, "Fabrication and Basic Characterization of a Piezoelectric Valveless Micro Jet Pump," Jpn. J. Appl. Phys., vol. 47, no. 11, pp. 8615-8618, Nov. 2008.

[2] V. T. Dau and T. X. Dinh, "Numerical study and experimental validation of a valveless piezoelectric air blower for fluidic applications," Sens. Actuators B Chem., vol. 221, pp. 1077-1083, Jul. 2015.

[3] V. T. Dau, T. X. Dinh, T. Katsuhiko, and S. Susumu, "A cross-junction channel valveless-micropump with PZT actuation," Microsyst. Technol., vol. 15, no. 7, pp. 1039-1044, May 2009.

[4] V. T. Dau, T. X. Dinh, and T. T. Bui, "Jet flow generation in a circulatory miniaturized system," Sens. Actuators B Chem., vol. 223, pp. 820-826, 2015.

[5] M. K. Araz, A. M. Tentori, and A. E. Herr, "Microfluidic multiplexing in bioanalyses.," J. Lab. Autom., vol. 18, no. 5, pp. 350-66, Oct. 2013.

[6] D. V. Dao, Van Thanh Dau, T. X. Dinh, and S. Sugiyama, "A Fully Integrated MEMS-Based Convective 3-DOF Gyroscope," in TRANSDUCERS 2007 - 2007 International Solid-State Sensors, Actuators and Microsystems Conference, 2007, pp. 1211-1214.

[7] V. T. Dau, T. X. Dinh, D. V. Dao, and S. Sugiyama, "Design and Simulation of a Novel 3-DOF MEMS Convective Gyroscope," IEEJ Trans. Sens. Micromachines, vol. 128, no. 5, pp. 219-224, May 2008.

[8] L. Y. Yeo, H.-C. Chang, P. P. Y. Chan, and J. R. Friend, "Microfluidic Devices for Bioapplications," Small, vol. 7, no. 1, pp. 12-48, Jan. 2011.

[9] H. K. K. Ma, H. C. C. Su, and W. F. F. Luo, "Investigation of a piezoelectric fan cooling system with multiple magnetic fans," Sens. Actuators Phys., vol. 189, pp. 356-363, Jan. 2013.

[10]P. Zhao, S. Portugal, and S. Roy, "Efficient needle plasma actuators for flow control and surface cooling," Appl. Phys. Lett., vol. 107, no. 3, p. $33501,2015$.

[11]Y. N. Kolomiets and V. V. Pervukhin, "Vortex focusing of ions produced in corona discharge," Talanta, vol. 110, pp. 39-45, 2013.

[12] V. T. Dau, T. X. Dinh, T. T. Bui, C.-D. Tran, H. T. Phan, and T. Terebessy, "Corona based air-flow using parallel discharge electrodes," Exp. Therm. Fluid Sci., vol. 79, pp. 52-56, 2016.

[13] V. T. Dau, T. X. Dinh, T. Terebessy, and T. T. Bui, "Bipolar corona discharge based air flow generation with low net charge," Sens. Actuators Phys., vol. 244, pp. 146-155, 2016.

[14] V. T. Dau, T. T. Bui, T. X. Dinh, and T. Terebessy, "Pressure sensor based on bipolar discharge corona configuration," Sens. Actuators Phys., vol. 237, pp. 81-90, Jan. 2016.

[15] V. T. Dau, T. X. Dinh, T. Terebessy, and T. T. Bui, "Ion Wind Generator Utilizing Bipolar Discharge in Parallel Pin Geometry," IEEE Trans. Plasma Sci., pp. 1-9, 2016.

[16] V. T. Dau, T. Otake, T. X. Dinh, D. V. Dao, and S. Sugiyama, "A multi axis fluidic inertial sensor," in Proceedings of IEEE Sensors, 2008, vol. 1, pp. 666-669.

[17] T. Shiozawa, V. T. Dau, D. V. Dao, H. Kumagai, and S. Sugiyama, "A dual axis thermal convective silicon gyroscope," in MicroNanomechatronics and Human Science, 2004 and The Fourth Symposium Micro-Nanomechatronics for Information-Based Society, 2004., 2004, pp. $1-6$.

[18] V. T. Dau, D. V. Dao, T. Shiozawa, and S. Sugiyama, "Simulation and Fabrication of a Convective Gyroscope," IEEE Sens. J., vol. 8, no. 9, pp. 1530-1538, Sep. 2008. 